PROCEEDINGS OF THE

AMERICAN MATHEMATICAL SOCIETY

Volume 137, Number 12, December 2009, Pages 4143-4155

S 0002-9939(09)09980-8

Article electronically published on July 13, 2009

\title{
CARLESON MEASURES FOR BERGMAN SPACES AND THEIR DUAL BEREZIN TRANSFORMS
}

\author{
BOO RIM CHOE, HYUNGWOON KOO, AND MICHAEL STESSIN
}

(Communicated by Franc Forstneric)

\begin{abstract}
We introduce the notion of weighted dual Berezin transforms and characterize Carleson measures for weighted Bergman spaces over the ball by a certain BMO property of their dual Berezin transforms.
\end{abstract}

\section{INTRODUCTION}

Let $\mathbb{B}=\mathbb{B}_{n}$ be the unit ball in the complex $n$-space $\mathbb{C}^{n}$. Throughout the paper we assume $n$ to be fixed. We use $V$ to denote the normalized volume measure on $\mathbb{B}$ and $V_{\alpha}$ with $\alpha>-1$ to denote the weighted measure defined by

$$
d V_{\alpha}(z)=c_{\alpha}\left(1-|z|^{2}\right)^{\alpha} d V(z)
$$

where the constant $c_{\alpha}$ is chosen so that $V_{\alpha}(\mathbb{B})=1$. For $0<p<\infty$ and $\alpha>-1$ the weighted Bergman space $A_{\alpha}^{p}(\mathbb{B})$ consists of all holomorphic functions $f$ on $\mathbb{B}$ such that

$$
\|f\|_{A_{\alpha}^{p}}:=\left\{\int_{\mathbb{B}}|f(z)|^{p} d V_{\alpha}(z)\right\}^{1 / p}<\infty .
$$

This norm turns $A_{\alpha}^{p}(\mathbb{B})$ into a Banach space for $1 \leq p<\infty$, but not for $0<p<1$. However, for $0<p<1, A_{\alpha}^{p}(\mathbb{B})$ is a complete metric space with the translationinvariant metric $(f, g) \mapsto\|f-g\|_{A_{\alpha}^{p}}^{p}$.

Recall that a finite positive Borel measure $\mu$ on $\mathbb{B}$ (hereafter we simply write $\mu \geq 0$ ) is said to be a Carleson measure for $A_{\alpha}^{p}(\mathbb{B})$ if $A_{\alpha}^{p}(\mathbb{B}) \subset L^{p}(\mu)$ or, equivalently (by the closed graph theorem), if there is a constant $C>0$ such that

$$
\int_{\mathbb{B}}|f(z)|^{p} d \mu(z) \leq C\|f\|_{A_{\alpha}^{p}}^{p}
$$

for all $f \in A_{\alpha}^{p}(\mathbb{B})$.

The first characterization of Carleson measures on $\mathbb{B}$ in terms of measures of Carleson sets was obtained by Hörmander $[\underline{\mathrm{H}}$ (in fact, this characterization was given for arbitrary smoothly bounded strongly pseudoconvex domains). Among

Received by the editors March 19, 2009.

2000 Mathematics Subject Classification. Primary 32A36; Secondary 32A18, 32A37.

Key words and phrases. Carleson measure, Bergman space, dual Berezin transform.

Part of this research was performed during the third author's visit to Korea University in 2008. He thanks the mathematics department of Korea University and the "Brain Pool" program for their hospitality and support. The first two authors were supported by the Korea Science and Engineering Foundation Grant funded by the Korean Government (KOSEF R01-2008-00020206-0).

(C)2009 American Mathematical Society Reverts to public domain 28 years from publication 4143 
other well-known characterizations of Carleson measures, we recall the one given in terms of Berezin transforms. Given a complex Borel measure $\mu$ on $\mathbb{B}$, the Berezin $\alpha$-transform $B_{\alpha} \mu$ of $\mu$ is a function on $\mathbb{B}$ defined by

$$
B_{\alpha} \mu(z)=\int_{\mathbb{B}}\left(\frac{1-|z|^{2}}{|1-\langle z, w\rangle|^{2}}\right)^{n+1+\alpha} d \mu(w), \quad z \in \mathbb{B}
$$

where $\langle z, w\rangle=\sum_{j=1}^{n} z_{j} \bar{w}_{j}$ denotes the Hermitian inner product on $\mathbb{C}^{n}$.

Given $\alpha>-1$ and $0<p<\infty$, it is well known that $\mu \geq 0$ is a Carleson measure for $A_{\alpha}^{p}(\mathbb{B})$ if and only if $B_{\alpha} \mu$ is bounded on $\mathbb{B}$; see [Zh, Theorem 2.25]. This characterization shows that the notion of Carleson measures depends on $\alpha$ but not on $p$. So, we may simply say " $\alpha$-Carleson measures" instead of "Carleson measures for $A_{\alpha}^{p}(\mathbb{B})$ ". Thus, if we set

$$
\|\mu\|_{\alpha}:=\sup _{\|f\|_{A_{\alpha}^{1}=1}} \int_{\mathbb{B}}|f(z)| d \mu(z),
$$

then $\mu$ is an $\alpha$-Carleson measure if and only if $\|\mu\|_{\alpha}<\infty$. Moreover, we have

$$
\|\mu\|_{\alpha} \approx\left\|B_{\alpha} \mu\right\|_{\infty}
$$

Here and elsewhere, the notation $X \approx Y$ means that $X / Y$ is bounded above and below by some positive constants depending on only the allowed parameters.

It is known that the Berezin $\alpha$-transform is bounded on $L^{2}\left(V_{\alpha}\right)$ for $\alpha>-1$; see [Zh, Theorem 2.10]. The adjoint operator of $B_{\alpha}$, when restricted to $L^{2}\left(V_{\alpha}\right)$, is easily computed and seen to extend to measures. That is, we define

$$
B_{\alpha}^{*} \mu(z)=\int_{\mathbb{B}}\left(\frac{1-|w|^{2}}{|1-\langle z, w\rangle|^{2}}\right)^{n+1+\alpha} d \mu(w), \quad z \in \mathbb{B}
$$

for $\alpha>-1$ and complex Borel measures $\mu$ on $\mathbb{B}$. We refer to the operator $B_{\alpha}^{*}$ as the dual Berezin $\alpha$-transform.

The purpose of this paper is to prove a characterization of Carleson measures by means of dual Berezin transforms. As far as we know, such observations have not appeared in the literature. In what follows $\mathcal{B}(\mathbb{B})$ denotes the Bloch space and $B M O_{G}^{\alpha}(\mathbb{B})$ denotes the $\alpha$-weighted Garcia BMO space. See Section 2 for precise definitions.

Theorem 1.1. Let $\alpha>-1$. Given $\mu \geq 0$, the following statements are equivalent:

(a) $\mu$ is an $\alpha$-Carleson measure;

(b) $B_{\alpha}^{*} \mu \in \mathcal{B}(\mathbb{B})$;

(c) $B_{\alpha}^{*} \mu \in B M O_{G}^{\alpha}(\mathbb{B})$.

We will also show that the conditions of Theorem 1.1 are equivalent to the hyperbolic Lipschitz condition introduced in Section 3 (see Theorem 3.4). Also, by means of the little Bloch space and VMO spaces, these equivalences carry over to vanishing $\alpha$-Carleson measures, meaning that the embedding $A_{\alpha}^{p}(\mathbb{B}) \subset L^{p}(\mu)$ is compact for some/all $p$ (see Theorem 3.5) .

Constants. Throughout the paper we use the same letter $C$ to denote various positive constants which may change at each occurrence. Variables indicating the dependency of constants $C$ will be often specified in parentheses or subscripts. 


\section{Prerequisites}

In this section we collect basic facts and definitions which we will need later. Details can be found in standard references such as $\underline{\mathrm{S}}$, Section 2.3] or [Zh, Section 1.2], unless otherwise specified.

2.1. Automorphism. For $z \in \mathbb{B}$, the standard automorphism $\varphi_{z}$ is given by

$$
\varphi_{z}(w)=\frac{z-P_{z} w-\sqrt{1-|z|^{2}} Q_{z} w}{1-\langle w, z\rangle}, \quad w \in \mathbb{B}
$$

where $P_{z}$ denotes the orthogonal projection of $\mathbb{C}^{n}$ onto the subspace generated by $z$ and $Q_{z}=I-P_{z}$. Then $\varphi_{z}$ is an automorphism(i.e. biholomorphic self-map) of $\mathbb{B}$. In fact $\varphi_{z}$ is an involution of $\mathbb{B}$ that exchanges the origin and the point $z$.

When one integrates a composite function with $\varphi_{z}$ against the measure $d V_{\alpha}(w)$, the expression

$$
\left(\frac{1-|z|^{2}}{|1-\langle z, w\rangle|^{2}}\right)^{n+1+\alpha}
$$

plays the role of the Jacobian. That is, we have the change-of-variables formula

$$
\int_{\mathbb{B}} h\left(\varphi_{z}(w)\right) d V_{\alpha}(w)=\int_{\mathbb{B}} h(w)\left(\frac{1-|z|^{2}}{|1-\langle z, w\rangle|^{2}}\right)^{n+1+\alpha} d V_{\alpha}(w)
$$

for $\alpha>-1$ and Borel functions $h$ on $\mathbb{B}$ whenever the integrals make sense. This can be verified by a routine calculation using the identity

$$
1-\left\langle\varphi_{z}(a), \varphi_{z}(b)\right\rangle=\frac{\left(1-|z|^{2}\right)(1-\langle a, b\rangle)}{(1-\langle a, z\rangle)(1-\langle z, b\rangle)}
$$

for all $a, b, z \in \mathbb{B}$.

2.2. Bergman metric. Given $z \in \mathbb{B}$ and $\zeta \in \mathbb{C}^{n}$, the Bergman metric $\beta(z, \zeta)$, modulo a constant factor, is given by the quadratic (in $\zeta$ )

$$
\beta^{2}(z, \zeta)=\frac{\left(1-|z|^{2}\right)|\zeta|^{2}+|\langle z, \zeta\rangle|^{2}}{\left(1-|z|^{2}\right)^{2}},
$$

and the corresponding Bergman (or hyperbolic) distance $d(z, w)$ between two points $z$ and $w$ in $\mathbb{B}$ is given by

$$
d(z, w)=\frac{1}{2} \log \frac{1+\left|\varphi_{z}(w)\right|}{1-\left|\varphi_{z}(w)\right|}
$$

see [Sh, p.301]. It is often convenient to use the pseudohyperbolic distance $\rho$ defined by

$$
\rho(z, w):=\left|\varphi_{z}(w)\right|
$$

We denote by $E_{r}(z)$ the pseudohyperbolic ball with center at $z$ and radius $r \in$ $(0,1)$. Since the Bergman metric is invariant under automorphisms, so is the (pseudo)hyperbolic distance.

Given $r \in(0,1)$ and $s$ real, there exists a constant $C=C(r, s)>0$ such that

$$
\left|\left(\frac{1-\langle a, z\rangle}{1-\langle a, w\rangle}\right)^{s}-1\right| \leq C \rho(z, w)
$$


for all $a, z, w \in \mathbb{B}$ with $\rho(z, w)<r$; see [Zh, Lemma 2.27] for details. As a consequence, we have

$$
1-|z|^{2} \approx 1-|w|^{2}
$$

for $z, w \in \mathbb{B}$ with $\rho(z, w)<r$.

2.3. Bloch space. Denote by $\partial_{j}=\frac{\partial}{\partial z_{j}}$ complex differentiation with respect to the $j$-th component of $z \in \mathbb{C}^{n}$. Also, let $\nabla=\left(\partial_{1}, \ldots, \partial_{n}\right)$ and $\bar{\nabla}=\left(\bar{\partial}_{1}, \ldots, \bar{\partial}_{n}\right)$. For $f \in C^{1}(\mathbb{B})$, we define

$$
Q f(z):=\sup _{\zeta \neq 0} \frac{|\langle\nabla f(z), \bar{\zeta}\rangle+\langle\bar{\nabla} f(z), \zeta\rangle|}{\beta(z, \zeta)}, \quad z \in \mathbb{B} .
$$

It is known $\mathrm{HY}$ that $Q$ is invariant under automorphisms in the sense that $Q(f \circ \varphi)=Q f \circ \varphi$ for all automorphisms $\varphi$ of $\mathbb{B}$.

We say that a function $f \in C^{1}(\mathbb{B})$ belongs to the Bloch space $\mathcal{B}(\mathbb{B})$ if the seminorm

$$
\|f\|_{\mathcal{B}}:=\sup _{z \in \mathbb{B}} Q f(z)
$$

is finite. Note that \|\|$_{\mathcal{B}}$ is also invariant under automorphisms. We also say that $f \in \mathcal{B}_{0}(\mathbb{B})$, the little Bloch space, if the additional boundary vanishing condition

$$
\lim _{|z| \rightarrow 1} Q f(z)=0
$$

is satisfied. We warn the reader that the terminology "(little) Bloch space" here is not standard; it usually refers to holomorphic functions in the literature.

2.4. Garcia BMO spaces. Let $\alpha>-1$. Given $f \in C(\mathbb{B})$, we say that $f$ belongs to the $\alpha$-weighted Garcia BMO space, $B M O_{G}^{\alpha}(\mathbb{B})$, if the semi-norm

$$
\|f\|_{G, \alpha}:=\sup _{a \in \mathbb{B}} \int_{\mathbb{B}}\left|f\left(\varphi_{a}(z)\right)-f(a)\right| d V_{\alpha}(z)
$$

is finite. We also say that $f \in V M O_{G}^{\alpha}(\mathbb{B})$ if the additional boundary vanishing condition

$$
\lim _{|a| \rightarrow 1} \int_{\mathbb{B}}\left|f\left(\varphi_{a}(z)\right)-f(a)\right| d V_{\alpha}(z)=0
$$

is satisfied.

2.5. Another characterization of Carleson measures. Given $\zeta \in \partial \mathbb{B}$ and $r>0$, we denote by $\Omega_{r}(\zeta)$ the Carleson set defined by

$$
\Omega_{r}(\zeta)=\{z \in \mathbb{B}:|1-\langle z, \zeta\rangle|<r\} .
$$

We recall here the well-known characterization of Carleson measures in terms of these Carleson sets.

For each fixed $\alpha>-1$, we have

$$
\|\mu\|_{\alpha} \approx \sup _{r, \zeta} \frac{\mu\left(\Omega_{r}(\zeta)\right)}{r^{n+1+\alpha}}
$$

details can be found in [CMc, Theorem 2.38]. 


\section{Carleson measures via dual Berezin transforms}

In this section we prove Theorem [1.1 as well as its compact analogue. In the course of the proof, the hyperbolic Lipschitz space of order 1 naturally comes into play. We denote by $\Lambda(\mathbb{B})$ the hyperbolic Lipschitz space of order 1 . Thus a function $f \in C(\mathbb{B})$ belongs to $\Lambda(\mathbb{B})$ if and only if the semi-norm

$$
\|f\|_{\Lambda}:=\sup _{z \neq a} \frac{|f(z)-f(a)|}{d(z, a)}
$$

is finite.

We begin with a simple observation.

Lemma 3.1. Given $\alpha>-1$, there is a constant $C=C(n, \alpha)>0$ such that

$$
C\|f\|_{G, \alpha} \leq\|f\|_{\Lambda} \leq\|f\|_{\mathcal{B}}
$$

for all $f \in C^{1}(\mathbb{B})$.

Proof. Let $f \in C^{1}(\mathbb{B})$ and fix $z \in \mathbb{B}$. Note that

$$
\left|f\left(\varphi_{a}(z)\right)-f(a)\right| \leq\|f\|_{\Lambda} d\left(\varphi_{a}(z), a\right)=\|f\|_{\Lambda} d(z, 0)
$$

for all $a, z \in \mathbb{B}$ by the automorphism invariance of $d$. Thus we have the first inequality with $C^{-1}=\int_{\mathbb{B}} d(z, 0) d V_{\alpha}(z)$. To prove the second inequality we first show that

$$
|f(z)-f(0)| \leq\left(\sup _{|w| \leq|z|} Q f(w)\right) d(z, 0)
$$

for $z \in \mathbb{B}$. Fix $z \neq 0$ and put $\zeta=z /|z|$. Note that

$$
\left|\frac{\partial}{\partial t}[f(t z)]\right|=|\langle\nabla f(t z), \bar{z}\rangle+\langle\bar{\nabla} f(t z), z\rangle| \leq Q f(t z)|z| \beta(t z, \zeta) .
$$

Thus, using the inequality $\beta(t z, \zeta) \leq\left(1-|t z|^{2}\right)^{-1}$ for $0<t<1$, we have

$$
\begin{aligned}
|f(z)-f(0)| & \leq \int_{0}^{1}\left|\frac{\partial}{\partial t}[f(t z)]\right| d t \\
& \leq\left(\sup _{|w| \leq|z|} Q f(w)\right) \int_{0}^{1} \frac{|z|}{1-|t z|^{2}} d t .
\end{aligned}
$$

Evaluating the last integral, we have (3.1). Now, as a consequence of (3.1), we have

$$
|f(z)-f(0)| \leq\|f\|_{\mathcal{B}} d(z, 0)
$$

for $z \in \mathbb{B}$. Thus, replacing $f$ by $f \circ \varphi_{w}$ and then $z$ by $\varphi_{w}(z)$, we obtain the second inequality by the automorphism invariance of \|\|$_{\mathcal{B}}$ and $d$.

We now prove a couple of lemmas to be used in the proof of Theorem 3.4 . We need the notion of the invariant gradient that allows us to compute $Q f$ explicitly. Given $f \in C^{1}(\mathbb{B})$ real and $z \in \mathbb{B}$, we denote by $|\widetilde{\nabla} f(z)|$ the invariant gradient at $z$, defined by

$$
|\widetilde{\nabla} f(z)|=\left|\nabla\left(f \circ \varphi_{z}\right)(0)\right| ;
$$

see [S, Section 3.4], where a slightly different constant factor is used. The next lemma explicitly expresses $Q f$ in terms of the invariant gradient. 
Lemma 3.2. If $f \in C^{1}(\mathbb{B})$ is real, then

$$
Q f(z)=2|\widetilde{\nabla} f(z)|
$$

for $z \in \mathbb{B}$.

Proof. Let $z \in \mathbb{B}$. For functions $f \in C^{1}(\mathbb{B})$, the equality

$$
\left|\nabla\left(f \circ \varphi_{z}\right)(0)\right|^{2}=\left(1-|z|^{2}\right)\left(|\nabla f(z)|^{2}-|\langle\nabla f(z), \bar{z}\rangle|^{2}\right)
$$

is known; see $[\underline{S}$, p. 28]. Also, for holomorphic functions $f$ on $\mathbb{B}$, a proof of the equalities

$$
Q f(z)^{2}=\left|\nabla\left(f \circ \varphi_{z}\right)(0)\right|^{2}=\left(1-|z|^{2}\right)\left(|\nabla f(z)|^{2}-|\langle\nabla f(z), \bar{z}\rangle|^{2}\right)
$$

can be found in $\left[\mathrm{Zh}\right.$, Theorem 3.1]. Since for a real-valued function $f \in C^{1}(\mathbb{B})$ we have $\overline{\bar{\nabla} f}=\nabla \bar{f}=\nabla f$, one may verify the lemma by the same proof.

We also need the following inequality. The symbol $\wedge$ below stands for the minimum.

Lemma 3.3. The inequality

$$
\int_{\mathbb{B}}\left(\frac{\left(1-|a|^{2}\right)\left(1-|b|^{2}\right)}{|1-\langle a, z\rangle|^{2}|1-\langle b, z\rangle|^{2}}\right)^{n+1+\alpha} d V_{\alpha}(z) \geq\left(\frac{1-|a|^{2} \wedge|b|^{2}}{|1-\langle a, b\rangle|^{2}}\right)^{n+1+\alpha}
$$

holds for all $\alpha>-1$ and $a, b \in \mathbb{B}$.

Proof. By symmetry we may assume that $|a| \leq|b|$. By the change-of-variables formula (2.1), the integral on the left-hand side of the inequality is equal to

$$
\int_{\mathbb{B}}\left(\frac{\left(1-|a|^{2}\right)}{\left|1-\left\langle a, \varphi_{b}(z)\right\rangle\right|^{2}}\right)^{n+1+\alpha} d V_{\alpha}(z) .
$$

Note that the integrand, as a function of $z$, is subharmonic. Thus, the above integral is greater than or equal to the value of the integrand at $z=0$, i.e.,

$$
\left(\frac{\left(1-|a|^{2}\right)}{|1-\langle a, b\rangle|^{2}}\right)^{n+1+\alpha}
$$

as required.

We are now ready to prove an expanded version of Theorem 1.1

Theorem 3.4. Let $\alpha>-1$. Given $\mu \geq 0$, the following statements are equivalent:

(a) $\mu$ is an $\alpha$-Carleson measure;

(b) $B_{\alpha}^{*} \mu \in \mathcal{B}(\mathbb{B})$;

(c) $B_{\alpha}^{*} \mu \in \Lambda(\mathbb{B})$;

(d) $B_{\alpha}^{*} \mu \in B M O_{G}^{\alpha}(\mathbb{B})$.

Moreover, the quantities

$$
\|\mu\|_{\alpha}, \quad \mu(\mathbb{B})+\left\|B_{\alpha}^{*} \mu\right\|_{\mathcal{B}}, \quad \mu(\mathbb{B})+\left\|B_{\alpha}^{*} \mu\right\|_{\Lambda}, \quad \mu(\mathbb{B})+\left\|B_{\alpha}^{*} \mu\right\|_{G, \alpha}
$$

are all comparable to one another. 
Proof. Fix $\mu \geq 0$ and put $f=B_{\alpha}^{*} \mu$ throughout the proof. By Lemma 3.1 we only need to show the implications (a) $\Longrightarrow$ (b) and (d) $\Longrightarrow($ a).

We first show that (a) implies (b). So, assume $\|\mu\|_{\alpha}<\infty$. It is sufficient to establish the estimate

$$
\|f\|_{\mathcal{B}} \leq C\|\mu\|_{\alpha}
$$

for some constant $C>0$ independent of $\mu$. Let $z \in \mathbb{B}$ and let $\mu \circ \varphi_{z}$ be the pullback measure defined by $\left(\mu \circ \varphi_{z}\right) E=\mu\left[\varphi_{z}(E)\right]$ for Borel sets $E \subset \mathbb{B}$. A standard argument shows that the action of $\mu \circ \varphi_{z}$ on continuous functions $g$ is given by

$$
\int_{\mathbb{B}} g \circ \varphi_{z} d \mu=\int_{\mathbb{B}} g d\left(\mu \circ \varphi_{z}\right)
$$

whenever the integrals make sense.

Let $a \in \mathbb{B}$. Since $\varphi_{z}$ is an involution, we have by (2.2) and (3.3) that

$$
\begin{aligned}
f \circ \varphi_{z}(a) & =\int_{\mathbb{B}}\left(\frac{1-|w|^{2}}{\left|1-\left\langle\varphi_{z}(a), w\right\rangle\right|^{2}}\right)^{n+1+\alpha} d \mu(w) \\
& =\int_{\mathbb{B}}\left(\frac{1-\left|\varphi_{z}(w)\right|^{2}}{\left|1-\left\langle\varphi_{z}(a), \varphi_{z}(w)\right\rangle\right|^{2}}\right)^{n+1+\alpha} d\left(\mu \circ \varphi_{z}\right)(w) \\
& =\int_{\mathbb{B}}\left(\frac{|1-\langle a, z\rangle|^{2}}{1-|z|^{2}} \cdot \frac{\left(1-|w|^{2}\right)}{|1-\langle a, w\rangle|^{2}}\right)^{n+1+\alpha} d\left(\mu \circ \varphi_{z}\right)(w) .
\end{aligned}
$$

Now, differentiating with respect to $a$ under the integral sign, we have

$$
\begin{aligned}
& \partial_{j}\left(f \circ \varphi_{z}\right)(a) \\
& =\frac{(n+1+\alpha)|1-\langle a, z\rangle|^{2(n+\alpha)} \overline{z_{j}}(\langle z, a\rangle-1)}{\left(1-|z|^{2}\right)^{n+1+\alpha}} \\
& \quad \times \int_{\mathbb{B}}\left(\frac{\left(1-|w|^{2}\right)}{|1-\langle a, w\rangle|^{2}}\right)^{n+1+\alpha} d\left(\mu \circ \varphi_{z}\right)(w) \\
& +(n+1+\alpha)\left(\frac{|1-\langle a, z\rangle|^{2}}{1-|z|^{2}}\right)^{n+1+\alpha} \\
& \quad \times \int_{\mathbb{B}} \frac{\overline{w_{j}}}{(1-\langle a, w\rangle)}\left(\frac{1-|w|^{2}}{|1-\langle a, w\rangle|^{2}}\right)^{n+1+\alpha} d\left(\mu \circ \varphi_{z}\right)(w)
\end{aligned}
$$

for each $j=1, \ldots, n$. Thus, substituting $a=0$, we obtain

$$
\nabla\left(f \circ \varphi_{z}\right)(0)=\frac{(n+1+\alpha)}{\left(1-|z|^{2}\right)^{n+1+\alpha}} \int_{\mathbb{B}}(\bar{w}-\bar{z})\left(1-|w|^{2}\right)^{n+1+\alpha} d\left(\mu \circ \varphi_{z}\right)(w) .
$$

Using (3.3) once more, we see that the integral in the right-hand side of the above is equal to

$$
\begin{aligned}
& \int_{\mathbb{B}}\left(\overline{\varphi_{z}(w)}-\bar{z}\right)\left(1-\left|\varphi_{z}(w)\right|^{2}\right)^{n+1+\alpha} d \mu(w) \\
= & \int_{\mathbb{B}}\left(\overline{\varphi_{z}(w)}-\bar{z}\right)\left(\frac{\left(1-|z|^{2}\right)\left(1-|w|^{2}\right)}{|1-\langle z, w\rangle|^{2}}\right)^{n+1+\alpha} d \mu(w) .
\end{aligned}
$$

It follows that

$$
\nabla\left(f \circ \varphi_{z}\right)(0)=\int_{\mathbb{B}} \frac{\overline{\varphi_{z}(w)}-\bar{z}}{|1-\langle z, w\rangle|^{2(n+1+\alpha)}} d \nu(w)
$$


where $d \nu(w)=(n+1+\alpha)\left(1-|w|^{2}\right)^{n+1+\alpha} d \mu(w)$. The inequality

$$
B_{n+1+2 \alpha} \nu(z) \leq(n+1+\alpha) B_{\alpha} \mu(z)
$$

is easily checked via (2.2).

Note that a straightforward calculation using (2.2) yields

$$
\begin{aligned}
\left|\varphi_{z}(w)-z\right|^{2} & =\left|\varphi_{z}(w)\right|^{2}+|z|^{2}-\left\langle\varphi_{z}(w), z\right\rangle-\left\langle z, \varphi_{z}(w)\right\rangle \\
& =\frac{\left(1-|z|^{2}\right)\left(|w|^{2}-|\langle w, z\rangle|^{2}\right)}{|1-\langle z, w\rangle|^{2}} \\
& \leq \frac{2\left(1-|z|^{2}\right)(1-|\langle w, z\rangle|)}{|1-\langle z, w\rangle|^{2}} \\
& \leq \frac{2\left(1-|z|^{2}\right)}{|1-\langle z, w\rangle|}
\end{aligned}
$$

for all $w \in \mathbb{B}$. It follows from (3.4) that

$$
|\widetilde{\nabla} f(z)|=\left|\nabla\left(f \circ \varphi_{z}\right)(0)\right| \leq \sqrt{2} \int_{\mathbb{B}} \frac{\left(1-|z|^{2}\right)^{1 / 2} d \nu(w)}{|1-\langle z, w\rangle|^{2(n+1+\alpha)+1 / 2}} .
$$

Also, note that by (3.5) and (1.1) $\nu$ is a $(n+1+2 \alpha)$-Carleson measure with $\|\nu\|_{n+1+2 \alpha} \leq C\|\mu\|_{\alpha}$ for some constant $C=C(n, \alpha)>0$. Thus, we have by (3.6) that

$$
|\widetilde{\nabla} f(z)| \leq \sqrt{2}\|\nu\|_{n+1+2 \alpha} \int_{\mathbb{B}} \frac{\left(1-|z|^{2}\right)^{1 / 2} d V_{n+1+2 \alpha}(w)}{|1-\langle z, w\rangle|^{2(n+1+\alpha)+1 / 2}} \leq C\|\mu\|_{\alpha}
$$

for some constant $C=C(n, \alpha)>0$. The last inequality holds because the integral above is finite (see [Zh, Theorem 1.12]). Since the estimate above is independent of $z \in \mathbb{B}$, we deduce (3.2) from Lemma 3.2. as required.

Now we show that (d) implies (a). So, assume $\|f\|_{G, \alpha}<\infty$. We need to establish the estimate

$$
C\|\mu\|_{\alpha} \leq \mu(\mathbb{B})+\|f\|_{G, \alpha}
$$

for some constant $C>0$ independent of $\mu$. In order to prove this we use (2.4). Since $\mu\left(\Omega_{r}(\zeta)\right) r^{-(n+1+\alpha)} \leq 4^{n+1+\alpha} \mu(\mathbb{B})$ for all $r \geq 1 / 4$ and $\zeta$, it is sufficient to show that there is a constant $C=C(n, \alpha)>0$ such that

$$
\sup _{\zeta \in \partial \mathbb{B}} \frac{\mu\left(\Omega_{r}(\zeta)\right)}{r^{n+1+\alpha}} \leq C \int_{\mathbb{B}}\left|f\left(\varphi_{a}(z)\right)-f(a)\right| d V_{\alpha}(z)
$$

for all $0<r<1 / 4$ and $a \in \mathbb{B}$ with $|a|=1-4 r$.

Fix $0<r<1 / 4$ and $a \in \mathbb{B}$ with $|a|=1-4 r$. Pick $\zeta \in \partial \mathbb{B}$ such that $a=(1-4 r) \zeta$. Note that we have, by (2.1) and Fubini's theorem,

$$
\begin{array}{rl}
\int_{\mathbb{B}} & f\left(\varphi_{a}(z)\right) d V_{\alpha}(z) \\
& =\int_{\mathbb{B}} f(z)\left(\frac{1-|a|^{2}}{|1-\langle a, z\rangle|^{2}}\right)^{n+1+\alpha} d V_{\alpha}(z) \\
& =\int_{\mathbb{B}} \int_{\mathbb{B}}\left(\frac{\left(1-|a|^{2}\right)\left(1-|w|^{2}\right)}{|1-\langle a, z\rangle|^{2}|1-\langle w, z\rangle|^{2}}\right)^{n+1+\alpha} d V_{\alpha}(z) d \mu(w) .
\end{array}
$$


Thus we have by Lemma 3.3 that

$$
\begin{aligned}
\int_{\mathbb{B}} \mid & f\left(\varphi_{a}(z)\right)-f(a) \mid d V_{\alpha}(z) \\
& \geq\left|\int_{\mathbb{B}}\left[f\left(\varphi_{a}(z)\right)-f(a)\right] d V_{\alpha}(z)\right| \\
& \geq \int_{\mathbb{B}}\left\{\left(\frac{\left(1-|a|^{2} \wedge|w|^{2}\right.}{|1-\langle a, w\rangle|^{2}}\right)^{n+1+\alpha}-\left(\frac{\left(1-|w|^{2}\right.}{|1-\langle a, w\rangle|^{2}}\right)^{n+1+\alpha}\right\} d \mu(w) \\
& =\int_{|w| \geq|a|} .
\end{aligned}
$$

Note that $|w| \geq|a|$ for $w \in \Omega_{r}(\zeta)$. Also, note that

$$
1-|w|^{2} \leq 2(1-|w|) \leq 2|1-\langle\zeta, w\rangle|<2 r=\frac{1-|a|}{2}
$$

for $w \in \Omega_{r}(\zeta)$. Accordingly, we have

$$
\begin{aligned}
\int_{|w| \geq|a|} & \geq \int_{\Omega_{r}(\zeta)}\left(\frac{(1-|a|}{|1-\langle a, w\rangle|^{2}}\right)^{n+1+\alpha}\left\{1-\left(\frac{1-|w|^{2}}{1-|a|}\right)^{n+1+\alpha}\right\} d \mu(w) \\
& \geq\left(1-\frac{1}{2^{n+1+\alpha}}\right)\left(\frac{1-|a|}{r^{2}}\right)^{n+1+\alpha} \mu\left(\Omega_{r}(\zeta)\right) \\
& =C \frac{\mu\left(\Omega_{r}(\zeta)\right)}{r^{n+1+\alpha}}
\end{aligned}
$$

where $C=4^{n+1+\alpha}\left(1-2^{-(n+1+\alpha)}\right)$. This yields (3.7), as asserted. The proof is complete.

We now turn to the characterization of the so-called vanishing Carleson measures. We say that $\mu \geq 0$ is a vanishing $\alpha$-Carleson measure for $A_{\alpha}^{p}(\mathbb{B})$ if the embedding $A_{\alpha}^{p}(\mathbb{B}) \subset L^{p}(\mu)$ is compact, meaning that every bounded sequence in $A_{\alpha}^{p}(\mathbb{B})$ has a convergent sequence in $L^{p}(\mu)$. The term "vanishing" comes from the following well-known characterizations:

$$
\begin{aligned}
\mu \text { is vanishing } \alpha \text {-Carleson for } A_{\alpha}^{p}(\mathbb{B}) & \Longleftrightarrow \lim _{|z| \rightarrow 1} B_{\alpha} \mu(z)=0 \\
& \Longleftrightarrow \lim _{r \rightarrow 0} \sup _{\zeta \in \partial \mathbb{B}} \frac{\mu\left(\Omega_{r}(\zeta)\right)}{r^{n+1+\alpha}}=0
\end{aligned}
$$

See [Zh, Theorem 2.26] for details where (3.9) is in a slightly different form. Also, see [CKS, Lemma 2.5] for a proof of (3.9) on the disk. So, the notion of vanishing Carleson measures is also independent of $p$. As in the case of $\alpha$-Carleson measures, we simply say "vanishing $\alpha$-Carleson measures" instead of "vanishing Carleson measures for $A_{\alpha}^{p}(\mathbb{B})$ ".

It is a well-known general phenomenon that once Carleson measures are characterized by a certain "big Oh" estimate, vanishing Carleson measures are then characterized by the corresponding "little Oh" estimate. Of course, $\mathcal{B}_{0}(\mathbb{B})$ and $V M O_{G}^{\alpha}(\mathbb{B})$ are natural candidates for "little Oh" subspaces of the $\mathcal{B}(\mathbb{B})$ and $B M O_{G}^{\alpha}(\mathbb{B})$, respectively, that appear in Theorem 3.4 For the "little Oh" subspace 
of $\Lambda(\mathbb{B})$, we introduce the space $\Lambda_{0}(\mathbb{B})$ consisting of functions $f$ such that

$$
\lim _{|a| \rightarrow 1} \sup _{\substack{z \in E_{r}(a) \\ z \neq a}} \frac{|f(z)-f(a)|}{d(z, a)}=0
$$

for some $r \in(0,1)$. Recall that $E_{r}(a)$ denotes the pseudohyperbolic ball with center at $a$ and radius $r \in(0,1)$.

We remark that if (3.10) holds for some $r$, then it is true for every $r \in(0,1)$. Indeed, if $r_{1}<r$, then the validity of (3.10) for $r_{1}$ is straightforward. Suppose that (3.10) holds for some $r$ and that $r<r_{1}<1$. Let $\epsilon>0$. Then we have some $\delta>0$ such that

$$
\frac{|f(z)-f(a)|}{d(z, a)}<\epsilon
$$

whenever $|a|>1-\delta$ and $0<d(z, a)<r$.

Note that by (2.3) we have a constant $c=c\left(n, r_{1}\right)>0$ such that for every pair of points $a$ and $z$ satisfying

$$
|a|>1-c \delta, \quad d(z, a)<r_{1},
$$

we have $|z|>1-\delta$. Now, let $a$ and $z$ satisfy (3.12), and let $\gamma$ be the Bergman geodesic which joins $a$ and $z$. Since the Bergman distance from any point of this geodesic to $a$ does not exceed $r_{1}$, the whole geodesic lies in the spherical annulus $\{1-\delta<|w|<1\}$ by (3.12), and, therefore, for any pair of points $\xi$ and $\eta$ on $\gamma$ with $0<d(\xi, \eta)<r$, we have $\frac{|f(\xi)-f(\eta)|}{d(\xi, \eta)}<\epsilon$ by (3.11). Fix a positive integer $N$ such that $r_{1}<N r$ and choose points $z^{0}=z, z^{1}, \ldots, z^{N}=a$ on $\gamma$ so that $d\left(z^{j}, z^{j+1}\right)=d(z, a) / N$ for each $j=0, \ldots, N-1$. Then, since $d(z, a) / N<r$, we have

$$
\frac{|f(z)-f(a)|}{d(z, a)} \leq \sum_{j=0}^{N-1} \frac{\left|f\left(z^{j}\right)-f\left(z^{j+1}\right)\right|}{N d\left(z^{j}, z^{j+1}\right)}<\epsilon .
$$

Since this holds for all $a$ and $z$ satisfying (3.12), we conclude that (3.10) with $r_{1}$ in place of $r$ also holds.

We are now ready to prove the "little Oh" version of Theorem 3.4

Theorem 3.5. Let $\alpha>-1$. Given $\mu \geq 0$, the following statements are equivalent:

(a) $\mu$ is a vanishing $\alpha$-Carleson measure;

(b) $B_{\alpha}^{*} \mu \in \mathcal{B}_{0}(\mathbb{B})$;

(c) $B_{\alpha}^{*} \mu \in \Lambda_{0}(\mathbb{B})$;

(d) $B_{\alpha}^{*} \mu \in V M O_{G}^{\alpha}(\mathbb{B})$.

Proof. Fix $\mu \geq 0$ and put $f=B_{\alpha}^{*} \mu$ throughout the proof.

First, we assume (a) and prove (b). Let $\nu$ be the measure introduced in the proof of Theorem 3.4. We see from (3.5) and (3.8) that $\nu$ is a vanishing $(n+1+2 \alpha)$ Carleson measure. Let $z \in \mathbb{B}$. We have by (3.6) that

$$
|\widetilde{\nabla} f(z)| \leq \sqrt{2} \int_{\mathbb{B}}\left|h_{z}(w)\right|^{2} d \nu(w)
$$

where $h_{z}(w)=\left(1-|z|^{2}\right)^{1 / 4}(1-\langle z, w\rangle)^{-(n+1+\alpha+1 / 4)}$. Note that the family $\left\{h_{z}\right\}_{z \in \mathbb{B}}$ is bounded in $A_{n+1+2 \alpha}^{2}(\mathbb{B})$ and converges to 0 uniformly on every compact subset of $\mathbb{B}$ as $|z| \rightarrow 1$. Thus $h_{z} \rightarrow 0$ weakly as $|z| \rightarrow 1$ in $A_{n+1+2 \alpha}^{2}(\mathbb{B})$. Now, since the 
embedding $A_{n+1+2 \alpha}^{2}(\mathbb{B}) \subset L^{2}(\nu)$ is compact, we see that the right-hand side of (3.13) tends to 0 as $|z| \rightarrow 1$. Thus (b) holds.

We show that (b) implies (c). Assume (b). Fix $r \in(0,1)$ and let $a \in \mathbb{B}$. We have

$$
\begin{aligned}
\sup _{\substack{z \in E_{r}(a) \\
z \neq a}} \frac{|f(z)-f(a)|}{d(z, a)} & =\sup _{0<|z|<r} \frac{\left|f \circ \varphi_{a}(z)-f(a)\right|}{d\left(\varphi_{a}(z), \varphi_{a}(0)\right)} \\
& =\sup _{0<|z|<r} \frac{\left|f \circ \varphi_{a}(z)-f(a)\right|}{d(z, 0)} .
\end{aligned}
$$

On the other hand, applying (3.1) (which is valid for any $C^{1}$-function) with $f \circ \varphi_{a}$ in place of $f$, we have

$$
\begin{aligned}
\left|f \circ \varphi_{a}(z)-f(a)\right| & \leq\left(\sup _{|w| \leq|z|} Q\left[f \circ \varphi_{a}\right](w)\right) d(z, 0) \\
& =\left(\sup _{w \in E_{|z|}(a)} Q f(w)\right) d(z, 0)
\end{aligned}
$$

for all $z \in \mathbb{B}$; the last equality comes from automorphism invariance of $Q$. Combining these observations, we obtain

$$
\sup _{\substack{z \in E_{r}(a) \\ z \neq a}} \frac{|f(z)-f(a)|}{d(z, a)} \leq \sup _{\substack{w \in E_{r}(a) \\ z \neq}} Q f(w) .
$$

Meanwhile, since $\lim _{|a| \rightarrow 1} Q f(a)=0$, we have $\sup _{w \in E_{r}(a)} Q f(w) \rightarrow 0$ as $|a| \rightarrow 1$ by (2.3). Now, taking the limit $|a| \rightarrow 1$ on both sides of the last displayed expressions above, we see that (b) holds.

We now assume (c) and show (d). Given $r \in(0,1)$ and $a \in \mathbb{B}$, write

$$
\int_{\mathbb{B}}\left|f\left(\varphi_{a}(z)\right)-f(a)\right| d V_{\alpha}(z)=\int_{r \mathbb{B}}+\int_{\mathbb{B} \backslash r \mathbb{B}} .
$$

For the integral over $r \mathbb{B}$, we have

$$
\begin{aligned}
\int_{r \mathbb{B}} & \leq\left(\sup _{0<|z|<r} \frac{\left|f\left(\varphi_{a}(z)\right)-f(a)\right|}{d(z, 0)}\right) \int_{r \mathbb{B}} d(z, 0) d V_{\alpha}(z) \\
& =\left(\sup _{\substack{z \in E_{r}(a) \\
z \neq a}} \frac{|f(z)-f(a)|}{d(z, a)}\right) \int_{r \mathbb{B}} d(z, 0) d V_{\alpha}(z) \\
& \rightarrow 0
\end{aligned}
$$

as $|a| \rightarrow 1$ (with $r$ fixed) by assumption. For the integral over $\mathbb{B} \backslash r \mathbb{B}$, we have

$$
\begin{aligned}
\int_{\mathbb{B} \backslash r \mathbb{B}} & \leq\|f\|_{\Lambda} \int_{\mathbb{B} \backslash r \mathbb{B}} d\left(\varphi_{a}(z), a\right) d V_{\alpha}(z) \\
& =\|f\|_{\Lambda} \int_{\mathbb{B} \backslash r \mathbb{B}} d(z, 0) d V_{\alpha}(z) \\
& \rightarrow 0
\end{aligned}
$$

as $r \rightarrow 1$, because $\int_{\mathbb{B}} d(z, 0) d V_{\alpha}(z)<\infty$. Thus, taking first the limit $|a| \rightarrow 1$ on the left-hand side of (3.14) and then the limit $r \rightarrow 1$, we see that (d) holds. 
Finally, the implication (d) $\Longrightarrow$ (a) follows from (3.7) and (3.9). The proof is complete.

\section{REMARKS}

Let $F: \mathbb{B}_{n} \rightarrow \mathbb{B}_{m}$ be a holomorphic map. It induces the composition operator $C_{F}$ given by

$$
C_{F} f=f \circ F
$$

for $f \in \mathcal{H}\left(\mathbb{B}_{m}\right)$, the space of holomorphic functions on $\mathbb{B}_{m}$. So, $C_{F}$ takes $\mathcal{H}\left(\mathbb{B}_{m}\right)$ into $\mathcal{H}\left(\mathbb{B}_{n}\right)$. By the standard measure-theoretic change of variables, it is easily seen that for $\alpha, \beta>-1, C_{F}$ is a bounded operator from $A_{\alpha}^{p}\left(\mathbb{B}_{m}\right)$ into $A_{\beta}^{p}\left(\mathbb{B}_{n}\right)$ if and only if the pullback measure $\mu_{F, \beta}:=V_{\beta} \circ F^{-1}$ is an $\alpha$-Carleson measure on $\mathbb{B}_{m}$.

For $n>m$ we denote by $\sigma_{n, m}$ the probability measure on the complex Grassmann manifold $\mathbb{G}_{m}\left(\mathbb{C}^{n}\right)$ which is invariant under the action of the unitary group. For an $m$-dimensional complex plane $s$ in $\mathbb{C}^{n}$ passing through the origin, we denote by $F_{s}$ the restriction of $F$ to $s \cap \mathbb{B}_{n}$. It is easy to see that

$$
B_{\alpha}^{*} \mu_{F, \beta}(z)=\int_{\mathbb{G}_{m}\left(\mathbb{C}^{n}\right)} B_{\alpha}^{*} \mu_{F_{s}, \beta}(z) d \sigma_{n, m}(s), \quad z \in \mathbb{B}_{m}
$$

where $\mu_{F_{s}, \beta}:=V_{\beta} \circ F_{s}^{-1}$ denotes the pullback measure on $\mathbb{B}_{m}$ induced by $F_{s}$ : $\mathbb{B}_{m} \rightarrow \mathbb{B}_{m}$.

In the case $n=m$, a result of Cima and Mercer [CM] states that $C_{F}: A_{\alpha}^{p}\left(\mathbb{B}_{n}\right) \rightarrow$ $A_{\alpha+n-1}^{p}\left(\mathbb{B}_{n}\right)$ is always bounded and, moreover, that

$$
\left\|C_{F}\right\| \leq C\left(\frac{1+|F(0)|}{1-|F(0)|}\right)^{\frac{\alpha+n+1}{p}}
$$

for some constant $C$ independent of $F$. This result has been extended to general cases that allow $n \neq m$. A result obtained for the ball setting by Koo and Smith $\underline{\mathrm{KS}}$ and Stessin and Zhu [SZ] or for the setting of more general domains by Poletsky and Stessin [PS] states that if $\beta \geq \alpha+m-1$, then $\mu_{F, \beta}$ is an $\alpha$-Carleson measure and, therefore, $C_{F}: A_{\alpha}^{p}\left(\mathbb{B}_{m}\right) \rightarrow A_{\beta}^{p}\left(\mathbb{B}_{n}\right)$ is bounded. In the case $n>m$, with help from the result by Cima and Mercer mentioned above, an application of Theorem 3.4 and relation (4.1) quickly yields another proof of such a result. We see a possible advantage of using dual Berezin transforms for the reason described below.

For a fixed $\alpha>-1$, the relation $\beta \geq \alpha+m-1$ gives a lower bound for $\beta$ that ensures boundedness of the composition operator from $A_{\alpha}^{p}\left(\mathbb{B}_{m}\right)$ into $A_{\beta}^{p}\left(\mathbb{B}_{n}\right)$. This estimate has been known to be sharp only in the case where $n=1$. In all other cases the problem of obtaining a sharp lower bound is still open. For instance, there is no known example of a holomorphic self-map $F$ of $\mathbb{B}_{2}$ such that $C_{F} A_{\alpha}^{2}\left(\mathbb{B}_{2}\right) \not \subset A_{\beta}^{2}\left(\mathbb{B}_{2}\right)$ with $\beta>\alpha+1 / 2$. Note that the dual Berezin transform approach allows us to express boundedness of the composition operator in terms of the rate of growth of weighted averages of the map. We believe that this point of view might be helpful in attempting to improve the lower bound or, hopefully, to obtain a sharp lower bound. 


\section{REFERENCES}

[CKS] B. R. Choe, H. Koo and W. Smith, Composition operators acting on holomorphic Sobolev spaces, Trans. Amer. Math. Soc. 355 (2003), 2829-2855. MR1975402 (2004e:47032)

[CM] J. Cima and P. Mercer, Composition operators between Bergman spaces on convex domains in $C^{n}$, J. Operator Theory 33 (1995), 363-369. MR.1354986 (96h:47036)

[CMc] C. Cowen and B. MacCluer, Composition operators on spaces of analytic functions, CRC Press, Boca Raton, FL, 1995. MR1397026 (97i:47056)

[HY] K. T. Hahn and E. H. Youssfi, M-harmonic Besov p-spaces and Hankel operators in the Bergman space on the ball in $C^{n}$, Manuscripta Math. 71 (1991), 67-81. MR.1094739 (92j:46047a)

[H] L. Hörmander, $L^{p}$ estimates for (pluri-)subharmonic functions, Math. Scand. 20 (1967), 65-78. MR0234002 (38:2323)

[KS] H. Koo and W. Smith, Composition operators between Bergman spaces of functions of several variables, Contemp. Math., 393, Amer. Math. Soc., Providence, RI, 2006, 123131. MR2198375 (2006k:47049)

[PS] E. A. Poletsky and M. I. Stessin, Hardy and Bergman spaces on hyperconvex domains and their composition operators, Indiana Univ. Math. J. 57 (2008), 2153-2201. MR.2463965

[Sh] B. V. Shabat, Introduction to complex analysis. Part II: Functions of several variables, Translations of Mathematical Monographs, 110, Amer. Math. Soc., Providence, RI, 1992. MR:1192135 (93g:32001)

[SZ] M. I. Stessin and K. Zhu, Composition operators on embedded disks, J. Operator Theory 56 (2006), 423-449. MR2282691 (2008a:47045)

[S] M. Stoll, Invariant potential theory in the unit ball of $C^{n}$, Cambridge University Press, Cambridge, 1994. MR.1297545 (96f:31011)

[Zh] K. Zhu, Spaces of holomorphic functions in the unit ball, Springer-Verlag, New York, 2005. MR2115155 (2006d:46035)

Department of Mathematics, Korea University, Seoul 136-713, Korea

E-mail address: cbr@korea.ac.kr

Department of Mathematics, Korea University, Seoul 136-713, Korea

E-mail address: koohw@korea.ac.kr

Department of Mathematics, The State University of New York, Albany, New York 12222

E-mail address: stessin@math.albany.edu 